\title{
Tecnologias lúdicas para adolescentes utilizadas por profissionais de saúde: revisão integrativa
}

Playful technologies for adolescents used by health professionals: an integrative review Tecnologías lúdicas para adolescentes utilizadas por profesionales de la salud: una revisión integradora

\author{
Marta Maria FranciscoI, Eliane Maria Ribeiro de Vasconcelos ${ }^{\text {II }}$, Maria Gorete \\ Lucena de Vasconcelos ${ }^{\mathrm{II}}$, Maria Auxiliadora Soares Padilha ${ }^{\mathrm{IV}}$, Ednaldo Cavalcante \\ de Araújov ${ }^{\mathrm{v}}$, Jones Sidnei Barbosa de Oliveira ${ }^{\mathrm{VI}}$
}

\begin{abstract}
Resumo: Objetivo: identificar as tecnologias lúdicas utilizadas pelos profissionais de saúde para adolescentes. Método: revisão Integrativa. As bases de dados foram: Lilacs, Bdenf, Cinahl, Adolec, Cuiden e Pepsic; SciELO, Cochrane library e Medline/Pubmed. Os descritores extraídos do DeCS e MESH, sendo a amostra final de cinco artigos originais. Período da busca de novembro de 2017 a janeiro de 2018. Resultados: os artigos apresentaram um jogo digital, um jogo papo reto, perguntas e respostas, o círculo de cultura, um dominó e um jogo de cartas. Estavam entre os anos de 2009 a 2018. O nível de evidência seis com rigor metodológico categoria A. Considerações finais: apesar dos artigos estarem voltados para adolescentes, ainda há uma necessidade de se desenvolver novas tecnologias lúdicas pelos profissionais de saúde, abrangendo outras doenças para esse público, sendo estes protagonistas do processo ensino-aprendizagem, mas também multiplicadores das ações de educação em saúde.
\end{abstract}

Descritores: Tecnologia; Adolescente; Aprendizagem

\footnotetext{
I Enfermeira. Mestre em Enfermagem. Universidade Federal de Pernambuco (UFPE). Recife, PE, Brasil. E-mail: marta_m_francisco@yahoo.com.br ORCID: https://orcid.org/0000-0001-8938-9179

${ }^{\text {II }}$ Enfermeira. Doutora em Enfermagem. Professora Associada IV do Departamento de Enfermagem da Universidade Federal de Pernambuco (UFPE). Recife, PE, Brasil. E-mail: emr.vasconcelos@gmail.com ORCID: https://orcid.org/0000-0003-3711-4194

III Enfermeira. Doutora em Enfermagem em Saúde Pública. Professora Associada IV do Departamento de Enfermagem da Universidade Federal de Pernambuco (UFPE). Recife, PE, Brasil. E-mail: mariagoretevasconcelos@gmail.com ORCID: https://orcid.org/0000-0001-7226-1646

IV Pedagoga. Doutora em Educação. Professora Associada I do Centro de Educação da Universidade Federal de Pernambuco (UFPE). Recife, PE, Brasil. E-mail: dorapadilha@gmail.com ORCID: https://orcid.org/0000-0001-7024-6522

$\checkmark$ Enfermeiro. Doutor em Ciências. Professor Adjunto II do Departamento de Enfermagem da Universidade Federal de Pernambuco (UFPE). Recife, PE, Brasil. E-mail: reuol.ufpe@gmail.com ORCID: https://orcid.org/0000-0002-1834-4544

VI Enfermeiro. Mestre em Enfermagem. Universidade Federal de Pernambuco (UFPE). Recife, PE, Brasil. E-mail: jonessidneyy@gmail.com ORCID: https://orcid.org/0000-0002-1170-2652
} 
Tecnologias lúdicas para adolescentes utilizadas por profissionais de saúde: revisão...। 2

Abstract: Objective: identify the playful technologies used by health professionals for adolescents.

Method: Integrative review. The databases were: Lilacs, Bdenf, Cinahl, Adolec, Cuiden and Pepsic; SciELO, Cochrane library and Medline / Pubmed. The descriptors extracted from DeCS and MESH, being the final sample of five original articles. Search period from November 2017 to January 2018. Results: the articles featured a digital game, a straight chat game, questions and answers, the culture circle, a domino and a card game. They were between the years 2009 to 2018. The level of evidence six with methodological rigor category A. Final considerations: despite the articles being aimed at teenagers, there is still a need to develop new playful technologies by health professionals, covering other diseases for this audience, these being protagonists of the teaching-learning process, but also multipliers of health education actions.

Descriptors: Technology; Adolescent; Learning

Resumen: Objetivo: identificar las tecnologías lúdicas utilizadas por los profesionales de la salud para adolescentes. Método: revisión integradora. Las bases de datos fueron: Lilacs, Bdenf, Cinahl, Adolec, Cuiden y Pepsic; SciELO, biblioteca Cochrane y Medline / Pubmed. Los descriptores extraídos de DeCS y MESH, siendo la muestra final de cinco artículos originales. Período de búsqueda de noviembre de 2017 a enero de 2018. Resultados: los artículos presentaron un juego digital, un juego de chat directo, preguntas y respuestas, el círculo cultural, un dominó y un juego de cartas. Fueron entre los años 2009 a 2018. Nivel de evidencia seis con categoría de rigor metodológico A. Consideraciones finales: aunque los artículos están dirigidos a adolescentes, aún existe la necesidad de desarrollar nuevas tecnologías lúdicas por parte de profesionales de la salud, que cubran otras enfermedades para este público, siendo estos protagonistas del proceso de enseñanza-aprendizaje, pero también multiplicadores de las acciones de educación para la salud.

Descriptores: Tecnología; Adolescente; Aprendizaje

\section{Introdução}

O lúdico é o estudo e prática para facilitação do aprendizado, e melhoria do desempenho por meio da interação, criação, uso e organização de processos e recursos tecnológicos. ${ }^{1}$ As mudanças provocadas pelo uso das tecnologias educacionais geram a necessidade de competências que, até então, não eram essenciais, mas deverão ser desenvolvidas pelos indivíduos. ${ }^{2}$

Neste contexto, a tecnologia educacional é um dos caminhos do processo educativo da criança e do adolescente e deverá ser inserida nas atividades de sala de aula, como ferramenta e não apenas como uma forma de automatizar esses processos, pois assim assume-se a produção de novos conhecimentos e não somente sua reprodução no processo ensino-aprendizagem. ${ }^{3}$ Nessa fase da adolescência, na qual 
ocorre à passagem da infância ao mundo adulto, é uma etapa de vida marcada por um complexo processo de crescimento e desenvolvimento biopsicossocial e espiritual, há uma maior necessidade de se desenvolver ferramentas por meio de novas tecnologias que alcancem o aluno dessa faixa etária, para que estes se sintam participativos, ativos sujeitos desse processo. ${ }^{4}$

Surgem nesse ciclo de vida, diversas peculiaridades como o desempenho de novos papéis sociais, a mudança na relação de dependência da família, além de escolhas de projetos de vida e dúvidas sobre as transformações biológicas ocorridas em decorrência da puberdade. ${ }^{4-5}$ Isso concorre para a exposição de situações de riscos e vulnerabilidades, que geram demandas de atenção à saúde e, podem implicar no desenvolvimento físico, cognitivo, social que incidem no processo de construção da cidadania.

Nessa fase, aumenta o interesse por novos conhecimentos e aprendizagens, impulsionando-os a buscar de maneira peculiar essas compreensões. ${ }^{5} \mathrm{O}$ uso de tecnologias, no dia a dia, instigam educadores e outros profissionais, ligados à área, para a investigação sobre como ocorrem a integração nos processos de aprendizagem. Dentre os vários recursos tecnológicos, presentes na escola, o jogo, é um dos que causa maior interesse e motivação aos estudantes. ${ }^{6}$

$\mathrm{Na}$ área da saúde, os jogos são considerados instrumentos educativos potencialmente capazes de contribuírem tanto para o desenvolvimento da educação como para a construção de conhecimentos em saúde. É visto como atividade divertida, estimulante, interativa, inovadora e ilustrativa, que responde à dupla tarefa de esclarecer dúvidas e facilitar a aprendizagem. O jogo, como tecnologia educacional, é uma das atividades que aperfeiçoa a criatividade dos envolvidos e propiciam ambientes prazerosos de aprendizado, necessários para emergir potenciais 
Tecnologias lúdicas para adolescentes utilizadas por profissionais de saúde: revisão...। 4

que facilitam a dinamização do contexto educativo e a construção do conhecimento a partir da realidade. ${ }^{6}$

A associação da tecnologia educacional com o lúdico requer propiciar uma aprendizagem utilizando brincadeiras e jogos. O ensino utilizando meios lúdicos cria um ambiente gratificante e atraente, propiciando estímulo para o desenvolvimento integral do indivíduo. ${ }^{7}$ Todas as ferramentas podem ser utilizadas, porém, a tecnologia lúdica, deverá ser avaliada na sua integração com as práticas educativas de modo a atender as necessidades e interesses da população alvo, e promover um momento de ensino-aprendizagem que seja coeso.

As múltiplas mídias desde o livro até a internet permitem a utilização de diversas linguagens e novas formas de comunicação. É crescente o número de escolas e centros de educação que estão usando ferramentas on-line e colaborativas para o aprendizado e busca de informações. ${ }^{8}$ Facilitadora do conhecimento, mediadora na construção de estruturas mentais, e como "ferramenta cognitiva" mediando o processo de aprendizagem. ${ }^{9}$

Pode-se enfatizar que as pessoas se encontram imersas em uma sociedade do conhecimento caracterizada pelo desenvolvimento de inovações das tecnológicas da informação e comunicação (TIC’s). O setor da saúde necessita de novas tecnologias que possam ampliar o conhecimento e empoderar a comunidade por meio da formação em saúde. Como exemplo, destacam-se as redes de tele saúde, o uso de softwares educacionais, a realidade virtual, a utilização dos computadores para simulações, o uso da internet e suas facilidades. ${ }^{10}$

O jogo como ferramenta educativa além de consistir em fontes de prazer e descoberta para os participantes constituem também forma de traduzir o contexto sócio histórico, refletido na cultura, o que contribui significativamente para o 
5 I Francisco MM, Vasconcelos EMR, Vasconcelos MGL, Padilha MAS, Araújo EC, Oliveira JSB

processo de construção crítica do conhecimento dos participantes. Aprender brincando enriquece as visões de mundo e estimula o relacionamento entre os pares, constituindo processo de troca de experiências e de socialização. ${ }^{11}$

As tecnologias lúdicas utilizadas em sala de aula como ferramenta da aprendizagem, associadas ao brincar na construção do conhecimento, agregam valores, motivação, troca de experiências, expõe dificuldades, interação e questionamentos, além de observar comportamentos antes não expostos. O educador, a princípio será o mediador, facilitador nesse processo. Posteriormente, esses alunos poderão caminhar sozinhos, na propagação desse conhecimento entre seus pares, nesse momento a educação em saúde acontece quando possibilita a transformação deste sujeito. ${ }^{12}$

Nessa perspectiva, a participação ativa desses adolescentes seus saberes, são elementos essenciais nas ações de promoção e prevenção da saúde. A utilização de tecnologias lúdicas para os adolescentes fomenta argumentações, reflexões e construção coletiva de conhecimento entre eles. ${ }^{13}$

Os profissionais de saúde devem atentar para a criação de estratégias de educação em saúde para que os adolescentes sejam capazes de conhecer as práticas que reiteram os aspectos históricos e sociais do processo saúde-doença. Propondo uma ação educativa que tenha caráter emancipatório de empoderamento, constituindo-se, dessa forma, um movimento de compreensão da realidade e de atenção à saúde no propósito de que algumas doenças quando diagnosticadas precocemente se obtém a cura, mas quando não é possível, se deve propor condições para melhorar a qualidade de vida. ${ }^{14}$ Nessa perspectiva, o objetivo dessa revisão integrativa foi identificar as tecnologias lúdicas utilizadas pelos profissionais de saúde para adolescentes. 
Tecnologias lúdicas para adolescentes utilizadas por profissionais de saúde: revisão...। 6

\section{Método}

Trata-se de um estudo do tipo revisão integrativa. Dentre outros métodos de revisão, a revisão integrativa é o mais amplo, sendo uma vantagem, pois permite a inclusão simultânea de estudos experimentais e não experimentais proporcionando uma compreensão completa do fenômeno analisado. Esse método também permite a combinação de dados de literatura teórica e empírica. Assim, o revisor pode elaborar uma revisão integrativa com diferentes finalidades e propósitos. ${ }^{15-16}$

Para a execução do estudo foram percorridas seis etapas distintas: 1 Elaboração do tema e da questão de pesquisa; 2 - Elaboração dos critérios de elegibilidade e busca dos estudos na literatura; 3 - Categorização dos estudos e extração dos dados; 4 - Avaliação dos estudos selecionados; 5 - Análise e interpretação dos resultados; 6 - Descrição dos resultados e discussão. ${ }^{17}$

Quanto aos critérios de elegibilidade, foram incluídos: artigos originais, publicados nos idiomas português, inglês ou espanhol. Não houve recorte temporal das publicações identificadas. Foram excluídos os trabalhos no formato de tese, dissertação, livro ou capítulo de livro, editorial, matéria de jornal, revisão integrativa ou sistemática da literatura, carta ao editor, estudo reflexivo, relato de experiência e nota prévia.

Vale salientar, que embora a biblioteca Cochrane library, tenha como principal objetivo as publicações dos artigos de Revisões Sistemáticas, foi incluída na estratégia de busca da seleção dos artigos, por ser uma biblioteca que também, armazena artigos originais. Isso ficou comprovado no último cruzamento realizado, em que foram encontrados 03 artigos originais, porém não foram incluídos na amostra final, por não responderem à questão de pesquisa. 
7 I Francisco MM, Vasconcelos EMR, Vasconcelos MGL, Padilha MAS, Araújo EC, Oliveira JSB

A estratégia de busca se deu em seis bases de dados: LILACS; BDENF; CINAHL; ADOLEC; CUIDEN e PePSIC; duas bibliotecas COCHRANE LIBRARY; e o portal PubMed que engloba a MEDLINE, uma biblioteca eletrônica SciELO, nas quais foram considerados como critério de seleção, apenas artigos originais, que estivessem publicados em revistas indexadas e que respondessem a questão de pesquisa. A busca foi realizada no período de novembro de 2017 a janeiro de 2018 . Os cruzamentos foram extraídos dos Descritores em Ciência da Saúde (DeCS) e do Medical Subject Headings (MeSH). Utilizaram-se os descritores em português: Tecnologia; Profissionais de Saúde; Adolescente; Aprendizagem. Inglês: Technology; Health Personal; Adolescent; Learning. Espanhol: Tecnología; Professional de Salud; Adolescente; Aprendizaje. Desse modo, o cruzamento entre os descritores supracitados foi realizado a partir da utilização do operador booleano “AND”.

Para essa pesquisa, foram realizados seis cruzamentos com os descritores do DeCS e MeSH:

1. Tecnologia AND Adolescente;

2. Aprendizagem AND Profissional de Saúde;

3. Aprendizagem AND Adolescente;

4. Tecnologia AND Profissional de Saúde AND Adolescente;

5. Aprendizagem AND Profissional de Saúde AND Adolescente;

6. Tecnologia AND Profissional de Saúde AND Adolescente AND Aprendizagem;

Desses cruzamentos foram identificados os quantitativos: 2.453 artigos no $1^{\text {o }}$ cruzamento; 917 artigos no $2^{\circ}$ cruzamento; 4.858 artigos no $3^{\text {o }}$ cruzamento; 424 artigos no $4^{\circ}$ cruzamento; 669 artigos no $5^{\circ}$ cruzamento; 41 artigos no $6^{0}$ cruzamento, 
Tecnologias lúdicas para adolescentes utilizadas por profissionais de saúde: revisão...। 8

perfazendo um total de 9.362 artigos encontrados nas diversas bases de dados, nos idiomas inglês, português ou espanhol (Quadro 1).

Quadro 1 - Seleção de artigos nas bases de dados por cruzamentos. Recife-PE, 2018.

\begin{tabular}{|c|c|c|c|c|c|c|c|}
\hline \multirow[b]{2}{*}{ BASES } & \multicolumn{6}{|c|}{ TOTAL POR CRUZAMENTOS } & \multirow[b]{2}{*}{ TOTAL GERAL } \\
\hline & $1^{0}$ & $2^{-}$ & $3^{\mathbf{0}}$ & $4^{\circ}$ & $5^{-}$ & $6^{\circ}$ & \\
\hline LILACS & 00 & 00 & 00 & 00 & 00 & 00 & 00 \\
\hline BDENF & 00 & 00 & 00 & 00 & 00 & 00 & 00 \\
\hline CINAHL & 690 & 175 & 1.923 & 224 & 08 & 01 & 3.021 \\
\hline ADOLEC & 00 & 00 & 00 & 00 & 00 & 00 & 00 \\
\hline CUIDEN & 69 & 111 & 32 & 107 & 03 & 00 & 322 \\
\hline $\begin{array}{c}\text { MEDLINE/ } \\
\text { PubMed }\end{array}$ & 467 & 46 & 373 & 55 & 611 & 37 & 1.589 \\
\hline PePSIC & 00 & 00 & 00 & 00 & 00 & 00 & 00 \\
\hline SciELO & 80 & 348 & 00 & 00 & 00 & 00 & 428 \\
\hline $\begin{array}{c}\text { COCHRANE } \\
\text { Library }\end{array}$ & 1.147 & 237 & 2.530 & 38 & 47 & 03 & 4.002 \\
\hline TOTAL & 2.453 & 917 & $\begin{array}{c}4.85 \\
8\end{array}$ & 424 & 669 & 41 & 9.362 \\
\hline
\end{tabular}

Após a identificação, realizou-se a seleção dos estudos primários, de acordo com a questão: quais as tecnologias lúdicas estão sendo utilizadas pelos profissionais de saúde para adolescentes? e os critérios de elegibilidade definidos. Todos os estudos identificados, por meio da estratégia de busca, foram inicialmente avaliados a partir da análise dos títulos, seguidos dos resumos. Nos casos em que os títulos e os resumos não se mostraram compreensíveis para definir a seleção inicial, procedeu-se à leitura na íntegra dos artigos selecionados. Conforme demonstra o fluxograma na Figura 1. 
9| Francisco MM, Vasconcelos EMR, Vasconcelos MGL, Padilha MAS, Araújo EC, Oliveira JSB

Figura 1 - Fluxograma com os resultados da seleção dos artigos selecionados conforme critérios de elegibilidade, Recife-PE, 2018.

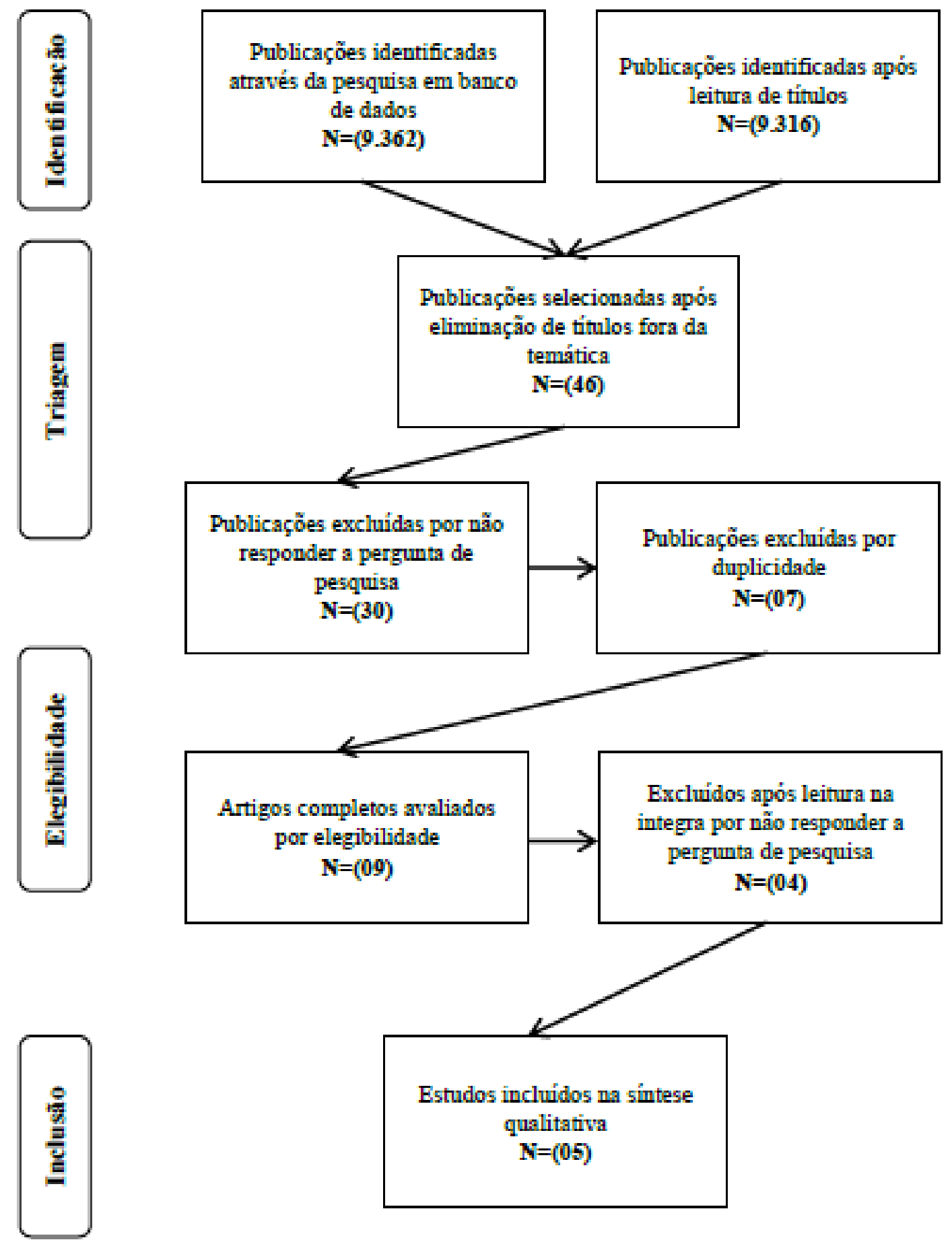

Fonte: Adaptado do modelo Prisma, 2009. 
Tecnologias lúdicas para adolescentes utilizadas por profissionais de saúde: revisão...। 10

Os estudos foram avaliados quanto ao nível de evidência pela abordagem metodológica que considera sete níveis segundo sua classificação: Nível I - Revisão Sistemática com ou sem Metanálise de todos os ensaios clínicos randomizados; Nível II Ensaio Clínico Randomizado, bem delineado; Nível III - Ensaio Clínico bem delineado, mas sem randomização; Nível IV - Estudos de Coorte e Caso-Controle; Nível V - Estudos originários de revisão sistemática de estudos descritivos e qualitativos; Nível VI - Evidências derivadas de um único estudo descritivo e qualitativo; Nível VII - Evidências oriundas de opiniões de autoridades e/ou relatório de comitês de especialistas. ${ }^{16-17}$

Os cinco artigos que compuseram a amostra final, submetidos à releitura, utilizou-se o formulário validado adaptado, composto por tópicos que contemplassem as principais questões dos artigos. ${ }^{17}$ Para o rigor metodológico das pesquisas aplicado na análise das publicações encontradas o instrumento adaptado do Critial Appraisal Skills Programm (CASP) este instrumento classifica os estudos como: de boa qualidade metodológica e viés reduzido (categoria A - 6 a 10 pontos), e com qualidade metodológica satisfatória (categoria B - no mínimo 5 pontos). Todos os cinco artigos encontraram-se na categoria A do rigor metodológico. Após essas análises, as informações relevantes foram reunidas em um quadrosíntese. ${ }^{17}$

As tecnologias mais encontradas foram as leves-duras, dentre elas um jogo de dominó, jogo de cartas, oficinas com círculo de cultura em que emergiu "o fanzine" todas essas tecnologias voltadas para os adolescentes.

\section{Resultados}

Dos cinco artigos finais, dois na SciELO (Sexualidade: reflexões teóricometodológicas ${ }^{18}$ hábitos alimentares saudáveis para adolescentes); ${ }^{19}$ dois na CINAHL (Educação em saúde em escola pública; ${ }^{20}$ DST/AIDS) ${ }^{21}$ e um na CUIDEN (Sexualidade para adolescentes). ${ }^{22}$

Em relação ao ano de publicação estavam entre os anos de 2009 a 2018. Quatro estudos foram produzidos no Brasil e um em Catalunya - Barcelona- Espanha. Em relação ao idioma, quatro estavam escritos em português e um em Espanhol. 
11 I Francisco MM, Vasconcelos EMR, Vasconcelos MGL, Padilha MAS, Araújo EC, Oliveira JSB

Os periódicos em que as publicações foram apresentadas, três estavam relacionados a área de enfermagem todos voltados aos conhecimentos dos adolescentes, a respeito de hábitos alimentares saudáveis, sexualidade e doenças sexualmente transmissíveis, dois abordavam o uso das tecnologias como ferramenta para o processo de ensino-aprendizagem, conteúdos voltados para educação como o círculo de cultura apoiado no modelo pedagógico de Paulo Freire, muito utilizado pelos profissionais de saúde quando são desenvolvidas ações de educação em saúde, principalmente na Atenção Básica. E o segundo abordava sobre a percepção dos profissionais da educação, pais e alunos em relação as tecnologias, como ferramenta do processo de ensino-aprendizagem. Os níveis de evidências de acordo com o sistema de classificação utilizado todos estavam no nível VI. Quanto ao rigor metodológico, todas as pesquisas obtiveram boa qualidade metodológica e viés reduzido, enquadrando-se nas pontuações de oito a dez.

O Quadro 2 mostra uma síntese das informações encontradas nos artigos selecionados. 


\begin{tabular}{|c|c|c|c|c|}
\hline Base/Autor/Ano & Título & Objetivo & Método / Nível de evidência & Resultados \\
\hline $\begin{array}{l}\text { SciELO/Souza et al/ } \\
2017 .{ }^{18}\end{array}$ & $\begin{array}{lr}\text { O jogo } & \text { como } \\
\text { estratégia } & \text { para } \\
\text { abordagem } & \text { da } \\
\text { sexualidade } & \text { com } \\
\text { adolescentes: } & \\
\text { reflexões teórico- } \\
\text { metodológicas }\end{array}$ & $\begin{array}{l}\text { Descrever o jogo Papo Reto } \\
\text { e refletir sobre suas bases } \\
\text { teórico-metodológicas }\end{array}$ & $\begin{array}{l}\text { Estudo analítico sobre o processo de } \\
\text { elaboração do jogo on-line Papo Reto para } \\
60 \text { adolescentes. Com faixa etária } \\
\text { predominante de } 18 \text { anos todos do ensino } \\
\text { médio de escolas públicas, foram } \\
\text { selecionados duas escolas: uma em Belo } \\
\text { Horizonte e a outra em São Paulo. } \\
\text { Nível de evidência: VI }\end{array}$ & 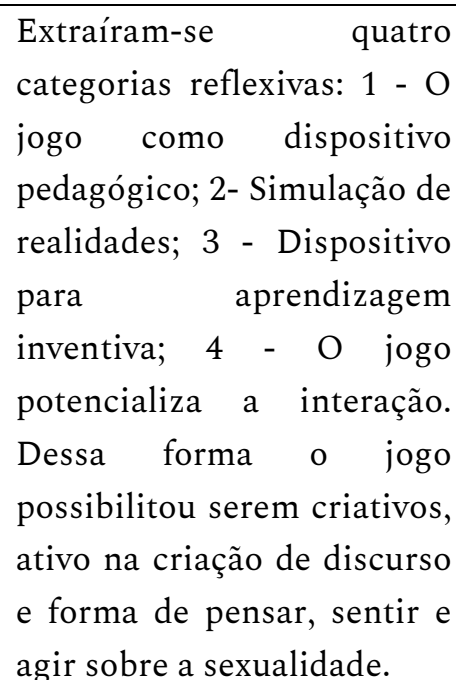 \\
\hline $\begin{array}{l}\text { SciELO/Carme Carrion } \\
\text { et al/2016. }{ }^{19}\end{array}$ & $\begin{array}{l}\text { Utilização de } \\
\text { celular para a } \\
\text { promoção de } \\
\text { hábitos saudáveis } \\
\text { em adolescentes. } \\
\text { Estudo com grupos } \\
\text { focais. }\end{array}$ & $\begin{array}{l}\text { Explorar as percepções, } \\
\text { valores, atitudes e } \\
\text { preferências } \\
\text { adolescentes, pais } \mathrm{e} \\
\text { professores sobre o uso das } \\
\text { tecnologias móveis para } \\
\text { promoção dos estilos de } \\
\text { vida saudáveis. }\end{array}$ & $\begin{array}{l}\text { Estudo exploratório, descritivo com } \\
\text { abordagem qualitativa. Amostragem por } \\
\text { conveniência, realizada em duas etapas: a } \\
\text { primeira a seleção das escolas; a segunda } \\
\text { a escolha dos } 30 \text { participantes que } \\
\text { formaram três grupos entre } 08 \text { e } 10 \\
\text { participantes. } \\
\text { Nível de evidência: VI }\end{array}$ & $\begin{array}{l}\text { Emergiram quatro } \\
\text { categorias dos grupos } \\
\text { focais: marco social e } \\
\text { cultural; adolescente e } \\
\text { saúde; papel da tecnologia } \\
\text { na vida dos adolescentes; } \\
\text { uso de tecnologia para } \\
\text { adquirir hábitos mais } \\
\text { saudáveis. Surgiram, } \\
\text { subcategorias atreladas ao } \\
\text { nexo entre adolescentes e } \\
\text { saúde. }\end{array}$ \\
\hline $\begin{array}{l}\text { CINAHL/Gubert et al/ } \\
2009 .{ }^{20}\end{array}$ & $\begin{array}{l}\text { Tecnologias } \\
\text { educativas no } \\
\text { contexto escolar: }\end{array}$ & 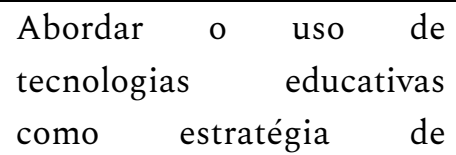 & $\begin{array}{l}\text { Estudo pesquisa-ação, na perspectiva } \\
\text { qualitativa, apoiado no Modelo } \\
\text { Pedagógico de Paulo Freire - Círculo de }\end{array}$ & $\begin{array}{lrr}\text { Nesse contexto, } & \text { os } \\
\text { profissionais de } & \text { saúde } \\
\text { devem produzir } & \text { novas }\end{array}$ \\
\hline
\end{tabular}




\begin{tabular}{|c|c|c|c|c|}
\hline & $\begin{array}{l}\text { estratégia de } \\
\text { educação em saúde } \\
\text { em escola pública } \\
\text { de Fortaleza-CE }\end{array}$ & $\begin{array}{l}\text { educação em saúde junto a } \\
\text { adolescentes no contexto } \\
\text { escolar. }\end{array}$ & $\begin{array}{l}\text { Cultura. Realizado com } 30 \text { alunos do } \\
\text { ensino médio de uma escola pública de } \\
\text { Fortaleza, na faixa etária de } 14 \text { a } 18 \text { anos. } \\
\text { Nível de evidência: VI }\end{array}$ & $\begin{array}{l}\text { tecnologias que favoreçam o } \\
\text { processo de educação em } \\
\text { saúde na prevenção das DST } \\
\text { e AIDS. Ao finalizar as } \\
\text { oficinas, emergiu a criação } \\
\text { de uma nova tecnologia } \\
\text { educacional "O Fanzine". }\end{array}$ \\
\hline $\begin{array}{l}\text { CINAHL/Barbosa et al } \\
/ 2010 .{ }^{21}\end{array}$ & $\begin{array}{l}\text { Jogo educativo } \\
\text { como estratégia de } \\
\text { educação em saúde } \\
\text { para adolescentes } \\
\text { na prevenção às } \\
\text { DST/AIDS }\end{array}$ & $\begin{array}{l}\text { Relatar o uso de jogos } \\
\text { educativos como estratégia } \\
\text { de educação em saúde para } \\
\text { adolescentes }\end{array}$ & $\begin{array}{l}\text { Estudo exploratório descritivo } \\
\text { desenvolvido em uma escola pública de } \\
\text { Fortaleza - CE, com } 85 \text { adolescentes entre } \\
\text { a faixa etária de } 14 \text { a } 19 \text { anos. O jogo } \\
\text { utilizado foi o dominó, juntamente com a } \\
\text { observação do participante, o protocolo } \\
\text { observacional, o pré e o pós-teste. } \\
\text { Nível de evidência: VI }\end{array}$ & $\begin{array}{l}\text { No pré-teste, percebeu o } \\
\text { desconhecimento dos } \\
\text { adolescentes no uso do } \\
\text { preservativo; No pós-teste, } \\
\text { constatou-se a eficácia da } \\
\text { ação } \\
\text { participativa. Ficou clara a } \\
\text { importância do jogo } \\
\text { educacional como um } \\
\text { processo de formação, } \\
\text { reflexão, interação e } \\
\text { participação grupal. Onde } \\
\text { estes puderam preencher as } \\
\text { lacunas e interagir com os } \\
\text { demais facilitando o } \\
\text { processo de ensino- } \\
\text { aprendizagem. }\end{array}$ \\
\hline
\end{tabular}


Tecnologias lúdicas para adolescentes utilizadas por profissionais de saúde: revisão...। 14

\begin{tabular}{|c|c|c|c|c|}
\hline $\begin{array}{l}\text { CUIDEN/ Souza et al / } \\
2018 .^{.2}\end{array}$ & $\begin{array}{lr}\text { Validação de } & \text { jogo } \\
\text { educativo } & \text { sobre } \\
\text { sexualidade } & \text { para } \\
\text { adolescentes } & \end{array}$ & $\begin{array}{l}\text { Validar jogo educativo } \\
\text { sobre sexualidade para } \\
\text { adolescentes }\end{array}$ & $\begin{array}{l}\text { Estudo metodológico de validação de } \\
\text { conteúdo e aparência. Desenvolvidos por } \\
\text { etapas: definição de temas; objetivos; } \\
\text { elaboração do jogo e validação de } \\
\text { conteúdo e aparência. } \\
\text { Nível de evidência: VI }\end{array}$ & $\begin{array}{l}\text { O jogo de cartas apresentou } \\
\text { um Índice de Validade de } \\
\text { Conteúdo e aparência de } \\
93 \% \text { o material está } \\
\text { aprovado como práticas } \\
\text { educativas de educação em } \\
\text { saúde para adolescentes. } \\
\text { Sendo essa mais uma } \\
\text { tecnologia educacional que } \\
\text { poderá ser utilizada por } \\
\text { profissionais de saúde para } \\
\text { adolescentes. }\end{array}$ \\
\hline
\end{tabular}

Quadro 2 - Caracterização dos estudos analisados segundo autor, base de dados e ano de publicação, título, objetivo, método, nível de evidência e principais resultados. Recife-PE, 2018. 
Tecnologias lúdicas para adolescentes utilizadas por profissionais de saúde: revisão integrativa I 15

\section{Discussão}

$\mathrm{Na}$ construção desta revisão foram encontrados vários artigos que abordavam sobre tecnologias educacionais, entretanto poucos voltados para ações com os adolescentes. Isso remete a um cenário de como está sendo trabalhada a educação em saúde com essa população.

O conhecimento produzido e publicado sobre a temática, tecnologias lúdicas utilizadas por profissionais de saúde, apresentou representativa participação da produção brasileira, das cinco pesquisas selecionadas, quatro foram produzidas no Brasil, publicadas em periódicos nacionais indexados. ${ }^{19,22}$ Tal fato demonstra que o Brasil tem participado na elaboração de conhecimentos sobre o uso de novas tecnologias no processo de ensino-aprendizagem.

A ludificação é o uso de técnicas de design que utilizam os jogos para, enriquecer contextos diversos, normalmente, não relacionados a jogo, mas aos conteúdos os quais os adolescentes poderão aprender por meio dessa ferramenta. Das publicações analisadas, todas retrataram a importância do lúdico no processo de ensino-aprendizagem para crianças e adolescentes, por motivar a reflexão, participação e discussão de forma interativa. ${ }^{18,22}$

A tecnologia móvel utilizada para conhecimento sobre hábitos alimentares saudáveis demonstrou o interesse dos adolescentes no desenvolvimento e mudanças desses hábitos e da autoestima pelo uso da tecnologia. ${ }^{19}$

A tecnologia se torna importante ferramenta educativa no processo em que estes adolescentes passam a ser protagonistas, expressando seus próprios questionamentos, anseios, dúvidas, mitos e verdades em situações problematizadoras, sem a exigência de determinado aprendizado ou condução do educador. A construção desse conhecimento provém de uma ação compartilhada, de modo comportamentais e que essa interação social torna-se condição indispensável para no processo de ensino-aprendizagem..$^{19}$

Nesse processo de aprendizagem e tecnologias, associados, os adolescentes poderão criar sua tecnologia, como exemplo, se teve esse estudo, em que os mesmos criaram "o fanzine" se 
Tecnologias lúdicas para adolescentes utilizadas por profissionais de saúde: revisão... | 16

sentindo protagonistas dessa ação-interação. ${ }^{20}$ Percebe-se a necessidade de criação de espaços de escuta nas escolas e serviços de saúde para adolescentes, que permita um vínculo de credibilidade estabelecido pelos profissionais da saúde e da educação, proporcionando assim escuta qualificada. ${ }^{21-22}$

A tecnologia educacional, embora desafiadora, pode executar uma ação entre a informação, debate, reflexão e participação grupal, mas os adolescentes devem compreender essa relação entre educação, processo criativo e inovador na construção da aprendizagem de forma que os saberes se completem à medida em que essa é colocada de forma dinâmica, destemida, comprometida e que haja engajamento. ${ }^{21}$

A principal característica pedagógica do jogo é favorecer uma atividade educativa pautada no referencial teórico com abordagem dialógica no processo de educação em saúde, buscando assim se distanciar da metodologia tradicional de ensino, na qual o educador apenas deposita conhecimentos na mente do educando. Diante do exposto, a dinâmica do jogo buscou incentivar a participação ativa dos adolescentes na construção de conhecimento. ${ }^{22}$

Essa participação ativa dos adolescentes na sondagem de seus saberes se constitui como elementos essenciais nas ações de promoção da saúde. O uso de jogos apresenta-se como instrumento do processo educativo em saúde que favorece a participação, debates e troca de experiências, sob a perspectiva crítica em relação à educação tradicional. ${ }^{18,22}$

No encontro entre tecnologias, jogos e educação, é factível uma educação autônoma pautada na dinâmica dos jogos, sem coibir um caráter libertário, espontâneo e insubordinado. A utilização de jogos educativos para adolescentes tem evidenciado que o trabalho lúdico e participativo fomenta argumentações, reflexões e construção coletiva de conhecimento entre si. Ao propor um jogo como atividade educativa, submete-se o caráter voluntário, próprio do jogo, aos objetivos do processo de aprendizagem, com o objetivo de torná-lo meramente um instrumento, ferramenta ou técnica de ensino. Ao subordinar-se a qualquer interesse que não 
ele próprio, o jogo pode perder o que melhor lhe define como princípio, ou seja, a rebeldia aos fins de utilidade e de produção. ${ }^{23}$

Nesse contexto os jogos são excelentes estratégias de educação em saúde, uma ferramenta singular para a promoção da saúde, portanto, é preciso aperfeiçoar essa prática junto aos profissionais de saúde, incentivando a elaboração de metodologias ativas que rompam paradigmas entre o que sabe e outro que não sabe, mas sim conscientizar que existem saberes diferentes que se completam à proporção que são inseridos como estratégias no processo de ensino aprendizagem. ${ }^{24}$

Embora haja controvérsias, autores reforçam a importância da tecnologia educacional no processo de ensino-aprendizagem, não para complementar, mas como parte essencial desse processo. O essencial é que o educador, assim como o educando possam, de forma prazerosa estar envolvidos nessa construção contínua de ensino-aprendizagem, utilizando essas ferramentas pedagógicas que se renovam e reinventam a medida que os protagonistas se permitem.

\section{Considerações finais}

A pesquisa vem corroborar para que os profissionais de saúde e da educação percebam a importância dessa tríade saúde-educação-tecnologia no processo de ensino-aprendizagem, sendo a escola um espaço crucial nessa interação, comunicação, diálogo e escuta. O adolescente escolar passa, a maior parte de seu tempo na escola, esse ambiente deve ser prazeroso, interativo, criativo, atrativo e receptivo.

Com os avanços tecnológicos e novos paradigmas nos modelos de ensino, as metodologias ativas favorecem esse campo abrangente por remeter a vários modelos de aprendizagens, na construção, desenvolvimento e aplicação de novas tecnologias educacionais em saúde e na educação. Na perspectiva de uma interação entre esses dois profissionais no 
Tecnologias lúdicas para adolescentes utilizadas por profissionais de saúde: revisão... | 18

processo de ações educativas em saúde, como é o caso do Programa de Saúde na Escola-PSE, que viabiliza a educação em saúde como estratégia de ensino-aprendizagem no desenvolvimento dos adolescentes como protagonistas sujeitos dessa ação. E, que possam estar envolvidos nesse processo de forma prazerosa, reflexiva, construtiva e interativa, adquirindo e levando conhecimentos para além da sala de aula.

Nesse contexto, faz-se necessário, a construção de tecnologias para promoção da saúde dos adolescentes abrangendo as diversas áreas da saúde, com o objetivo de levar conhecimento e empoderamento nessa fase importante de descobertas, desenvolvimento e crescimento dos adolescentes, concomitante ao processo de ensino-aprendizagem.

As limitações do estudo se referem à amostra não ter utilizado o critério de qualidade duplo-independente na seleção e extração dos artigos. Porém, conseguiu responder e atingir o objetivo proposto na pesquisa.

\section{Referências}

1. Viva MAA, Vianna PBM. Entre nativos e imigrantes digitais: um estudo na educação superior. Rev Cesuca Virtual [Internet]. 2013 [acesso em 2018 jan 12];1(2). Disponível em: http://ojs.cesuca.edu.br/index.php/cesucavirtual/article/view/584

2. Martins RX, Fátima FV. A implantação do Programa Nacional de Tecnologia Educacional (ProInfo): revelações de pesquisas realizadas no Brasil entre 2007 e 2011. Rev Bras Estud Pedagog [Internet]. 2015 [acesso em 2017 nov 08];(96)242:112-28. Disponível em: http://www.scielo.br/scielo.php?pid=S2176$66812015000100112 \&$ script $=$ sci_abstract\&tlng=pt

3. Almeida MEBA, Valente JA. Tecnologias e currículo: trajetórias convergentes e divergentes? São Paulo: Paulus; 2011.

4. World Health Organization (WHO). Young people's health - a challenge for society. Report of a who study group on young people and health for all. Tech Rep Ser 731. Geneva: WHO; 1986.

5. Brasil. Lei no 8.069, de 13 de julho de 1990. Dispõe sobre o Estatuto da Criança e do Adolescente e dá outras providências. Brasília (DF): Presidência da República; 1990. 
6. Kishimoto TM. Jogos, brinquedos e brincadeiras do Brasil. Espac Blanco, Ser Indagaciones [Internet], 2014 [acesso em 2018 mar 08]; (24): 10-22. Disponível em: http://www.espaciosenblanco.unicen.edu.ar/pdf/numerorosanterior/Revista_Espacios_en_Blanco_N24.pd $\mathrm{f}$

7. Alves L, Bianchin MA. O jogo como recurso de aprendizagem. Rev Psicopedag. 2010;27(83):282-7.

8. Blakely G, Skirton H, Cooper S, Allum P, Nelmes P. Educational gaming in the health sciences: systematic review. J Adv Nurs. 2009;65(2):259-69.

9. Gubert FA, Vieira NFC, Pinheiro PNC, Oriá MOB, Almeida PC, Araújo TS. Tradução e validação da escala Parent-adolescent Communication Scale: tecnologia para prevenção de DST/HIV. Rev Latinoam Enferm [Internet]. 2013 [acesso em 2018 jan 10];21(4):1-8. Disponível: http://www.scielo.br/pdf/rlae/v21n4/pt_0104-1169-rlae-21-04-0851.pdf

10. Silveira RCP, Nagata M, Maia LLQGN, Araújo A, Cavalcante RB. uso de tecnologias da informação e comunicação na educação em saúde de adolescentes escolares. J. Health Inform [Internet]. 2012 [acesso em 2018 jan 11];4(4):182-6. Disponível em: https://www.researchgate.net/publication/281784182_Uso_de_Tecnologias_da_Informacao_e_Comunicac ao_na_educacao_em_saude_de_adolescentes_escolares

11. Malaquias MS, Ribeiro SS. A Importância do lúdico no processo de ensino-aprendizagem no desenvolvimento da infância. Psicologado [Internet]. 2013 [acesso em 2017 abr 02];7(1):16-1. Disponível em: https:/psicologado.com/atuacao/psicologia-escolar/a-importancia-do-ludico-no-processo-de-ensinoaprendizagem-no-desenvolvimento-da-infancia

12. Lavinas L, Veiga A. Desafios do modelo brasileiro de inclusão digital pela escola. Cad Pesqui [Internet]. 2013 [acesso em 2017 dez 07];43(149):542-69. Disponível em: http://publicacoes.fcc.org.br/ojs/index.php/cp/article/view/2665

13. Sousa MG, Oliveira EML, Coelho MMF, Miranda KCL, Henriques ACPT, Cabral RL. Validação de jogo educativo sobre sexualidade para adolescentes. Rev Pesqui Cuid Fundam [Internet]. 2018 [acesso em 2017 nov 08];10(1):203-9. Disponível em: http://www.seer.unirio.br/index.php/cuidadofundamental/article/view/6030

14. Ribeiro MDA, Oliveira SB, Filgueiras MC. Pós-alta em hanseníase: uma revisão sobre qualidade de vida e conceito de cura. Saúde (Santa Maria) 2015;41(1):09-18.

15. Souza MT, Silva MD, Carvalho R. Revisão integrativa: o que é e como fazer. Einstein. 2010;8(1):102-6.

16. Ganong LH. Integrative reviews of nursing research. Res Nurs Health. 1987;10(1):1-11.

17. Ursi ES, Galvão CM. Prevenção de lesões de pele no perioperatório: revisão integrativa da literatura. Rev Latinoam Enferm 2006;14(1):124-31. 
18. Souza V, Gazzinelli MF, Soares AN, Fernandes MM, Oliveira RNG, Fonseca RMGS. The game as strategy for approach to sexuality with adolescents: theoretical-methodological reflections. Rev Bras Enferm [Internet]. 2017 [acesso em 2017 nov 08];70(2):376-83. Disponível em: http://dx.doi.org/10.1590/0034-7167-2016-0043

19. Carrion C, Arroyo ML, Castell C, Puigdomènech E, Gómez SF, Domingo L, et al. Utilización del teléfono móvil para el fomento de hábitos saludables en adolescentes. Estudio con grupos focales. Rev Esp Salud Pública. 2016;(90)3:1-11.

20. Gubert FA, Santos ACL, Aragão KA, Pereira DCR, Vieira NFC, Pinheiro PNC. Tecnologias educativas no contexto escolar: estratégia de educação em saúde em escola pública de Fortaleza-CE. Rev Eletrônica Enferm [Internet]. 2009 [acesso em 2017 dez 10];11(1):165-72. Disponível em: https://www.fen.ufg.br/revista/v11/n1/pdf/v11n1a21.pdf

21. Barbosa SM, Dias FLA, Pinheiro AKB, Pinheiro PNC, Vieira NFC. Jogo educativo como estratégia de educação em saúde para adolescentes na prevenção às DST/AIDS. Rev Eletrônica Enferm [Internet]. 2010 [acesso em 2018 jan 10];12(2):337-41. Disponível em: https://www.fen.ufg.br/fen_revista/v12/n2/v12n2a17.htm

22. Sousa MG, Oliveira EML, Coelho MMF, Miranda KCL, Henriques ACPT, Cabral RL. Validação de jogo educativo sobre sexualidade para adolescentes. Rev Pesqui Cuid Fundam [Internet]. 2018 [acesso em 2017 nov 08];10(1):203-9. Disponível em: http://www.seer.unirio.br/index.php/cuidadofundamental/article/view/6030

23. Pires MRGM, Guilhem D, Göttems LBD. Jogo (in)dica-SUS: estratégia lúdica n a aprendizagem sobre o Sistema Único De Saúde. Texto \& Contexto Enferm. 2013;22(2):379-88.

24. Yonekura T, Soares CB. O jogo educativo como estratégia de sensibilização para coleta de dados com adolescentes. Rev Latinoam Enferm [Internet]. 2010 [acesso em 2017 dez 10];18(5):1-7. Disponível em: http://www.scielo.br/pdf/rlae/v18n5/pt_18.pdf

\section{Autor correspondente}

Marta Maria Francisco

E-mail: marta_m_francisco@yahoo.com.br

Endereço: Rua Engenheiro Moacyr Parahyba, 230, Iputinga.

Recife, Pernambuco, Brasil.

CEP: 50800-320 
21 I Francisco MM, Vasconcelos EMR, Vasconcelos MGL, Padilha MAS, Araújo EC, Oliveira JSB

\section{Contribuições de Autoria}

\section{1 - Marta Maria Francisco}

concepção e planejamento da revisão, obtenção, análise e interpretação dos dados, redação e revisão crítica.

\section{2 - Eliane Maria Ribeiro de Vasconcelos}

Planejamento da revisão, análise e interpretação dos dados, revisão crítica.

\section{3 - Maria Gorete Lucena de Vasconcelos}

Planejamento da revisão, análise e interpretação dos dados, revisão crítica.

4 - Maria Auxiliadora Soares Padilha

Redação e revisão crítica.

5 - Ednaldo Cavalcante de Araújo

Redação e revisão crítica.

6 - Jones Sidnei Barbosa de Oliveira

Análise e interpretação dos dados, redação e revisão crítica.

\section{Como citar este artigo}

Francisco MM, Vasconcelos EMR, Vasconcelos MGL, Padilha MAS, Araújo EC, Oliveira JSB. Tecnologias lúdicas para adolescentes utilizadas por profissionais de saúde: revisão integrativa. Rev. Enferm. UFSM. 2020 [Acesso em: Anos Mês Dia]; vol.10 e31: 1-21. DOI: https://doi.org/10.5902/2179769237050 